Journal of Advanced Research in Fluid Mechanics and Thermal Sciences

\title{
Mathematical Modeling of Unsteady Solute Dispersion in Bingham Fluid Model of Blood Flow Through an Overlapping Stenosed Artery
}

\author{
Siti Nurulaifa Mohd ZainulAbidin ${ }^{1}$, Zuhaila Ismail $^{1,},{ }^{*}$ Nurul Aini Jaafar ${ }^{1}$ \\ 1 Department of Mathematical Sciences, Faculty of Science, Universiti Teknologi Malaysia, 81310 Skudai, Johor, Malaysia
}

\begin{tabular}{ll}
\hline ARTICLE INFO & ABSTRACT \\
\hline $\begin{array}{l}\text { Article history: } \\
\text { Received } 19 \text { May } 2021\end{array}$ & $\begin{array}{l}\text { An artery narrowing referred to as atherosclerosis or stenosis causes a reduction in the } \\
\text { diameter of the artery. When blood flow through an artery consists of stenosis, the } \\
\text { Accepted } 3 \text { September } 2021\end{array}$ \\
$\begin{array}{l}\text { Available online } 4 \text { October } 2021 \\
\text { issue of solute dispersion is more challenging to solve. A mathematical model is } \\
\text { developed to examine the unsteady solute dispersion in an overlapping stenosed artery } \\
\text { portraying blood as Bingham fluid model. The governing of the momentum equation } \\
\text { and the constitutive equation is solved analytically. The generalized dispersion model } \\
\text { is imposed to solve the convective-diffusion equation and to describe the entire } \\
\text { dispersion process. The dispersion function at steady-state decreases at the center of } \\
\text { an artery as the stenosis height increase. A reverse behavior is shown at an unsteady- } \\
\text { state. As the plug core radius, time and stenosis height increase, the dispersion function } \\
\text { decreases at the center of an artery. There is a high amount of red blood cells at the } \\
\text { center of the artery but no influences near the wall. Hence, this model is useful in } \\
\text { transporting the drug or nutrients to the targeted stenosed region in the treatment of } \\
\text { fluid; Overlapping stenosis; Steady flow }\end{array}$ & \begin{tabular}{l} 
diseases and in understanding many physiological processes. \\
\hline
\end{tabular}
\end{tabular}

\section{Introduction}

The World Health Organization (WHO) reported that coronary artery disease (CAD) is the pioneer cause of death with $16 \%$ of the world's estimated deaths [1]. In Malaysia, this is a similar situation as CAD is ranked as the first cause of death with $15 \%$ of the total population. The major cause of cardiovascular disorders is atherosclerosis [2]. Atherosclerosis is a chronic inflammatory process at the artery walls which leads to wall thickening, lumen narrowing and blood flow obstruction. The irregular and abnormal growth in the arterial wall is caused by stenosis or atherosclerosis due to the massive accumulation of cholesterol, fats and abnormal growth of tissue. According to Shah [3] and Mishra and Siddiqui [4], when blood flows through an artery consists of stenosis in certain regions, the issue of solute dispersion is more difficult to solve.

Many scholars study extensively the solute dispersion in a solvent as it has many uses in different fields of science and engineering [5, 6]. Taylor [7] was the pioneer who investigated solute dispersion and showed that, as the solute flows slowly in a straight tube into a solvent, the inclusion combination

\footnotetext{
* Corresponding author.

E-mail address: zuhaila@utm.my

https://doi.org/10.37934/arfmts.87.3.134147
} 
of molecular diffusion and the velocity variance over the cross-section will eventually lead the solute diffusing with molecular diffusivity given $D_{\text {eff }}=a^{2} w_{m}{ }^{2} / 48 D_{m}$, where $D_{m}$ is the molecular diffusivity, $w_{m}$ is the mean axial velocity and $a$ is the mean axial velocity and $a$ is the radius of the tube. Aris [8] executing the method of moments reported that the dispersion theory of Taylor is only valid when $D_{e f f} \geq D_{m}$ and thereby enhanced the dispersion theory by including the effect of axial molecular diffusion $D_{e f f}=D_{m}+a^{2} w_{m}{ }^{2} / 48 D_{m}$. Thereafter, Gill [9] simplified Taylor-Aris's work by suggesting a series expansion on the mean concentration to define the distribution of the local concentration which is applicable for all time. Gill and Sankarasubramanian [10] improved the work using the generalized dispersion model (GDM) which defines the entire process of dispersion in a series solution. Their outcomes also verified Ananthakrishnan [11]'s finding that the concept of Taylor-Aris dispersion applies over time surpass $0.5\left(a^{2} / D_{m}\right)$.

Shahzadi and Bilal [12] using a Newtonian fluid through a bifurcated mild stenosed artery used copper and its oxide as a drug to minimize the lesions of the atherosclerotic artery. Haghighi et al., [13] deduced that increasing the stenosis size effect in decreasing velocity of the blood. Meanwhile, Das et al., [14] analyzed the dispersion of solute by considering the blood as Casson fluid using a numerical method of IBM-MAC in a mild-shaped stenosed artery. They revealed that in the distribution of solute, stenosis plays a significant role. Chakravarty and Mandal [15] identified that more cardiovascular diseases are caused by overlapping stenosis instead of mild stenosis. Ahmed and Nadeem [16] using a Carreau fluid analyzed a blood flow through an inclined artery with overlapping stenosis. Bhatnagar and Srivastav [17] analyzed the influence of time and slip velocity on the flow of blood with overlapping stenosis. Hossain and Haque [18] and Freidoonimehr et al., [19] claimed that the blockage of a coronary artery with stenosis is highly determined by its shape and degree of stenosis. Meanwhile, [20] using a Newtonian fluid ascribed that the size or the severity of the stenosis has a great impact on the flow field (velocity distribution) and wall shear stress distribution of stenosis.

In the present analysis, a mathematical model is developed to analyze mathematically the unsteady solute dispersion in a steady flow of blood represented by the Bingham-Plastic fluid model utilizing the method of GDM in an overlapping stenosed artery. The study may be relevant in understanding many physiological processes that involve injecting a quantity of solute into the bloodstream and dispersion of drugs or nutrients in the circulatory system. The goal is to investigate the influence of physical parameters such as stenosis height and plug core radius on the solute dispersion in the solvent. This studied extends the validity of the results for a time smaller than $0.5\left(a^{2} / D_{m}\right)$.

\section{Problem Formulation}

The solute dispersion in blood flow is assumed to be a steady, laminar, axially symmetrical and fully developed uni-directional flow of blood as in Figure 1. The blood flow in the axial direction is portrayed as a viscous incompressible fluid.

\subsection{Governing Equations}

The cylindrical polar coordinates $(\bar{r}, \bar{\psi}, \bar{z})$ where $\bar{r}$ and $\bar{z}$ indicates the radial and axial coordinates and $\bar{\psi}$ is the azimuthal angle is considered. This works will ignore the fluid velocity in $\bar{r}$ direction as its magnitude is negligibly small and only accounts in a $\bar{z}$ direction. Hence, $\bar{u}_{\bar{r}}=\bar{u}_{\bar{\psi}}=0$ [21]. Due to the axial symmetry, the velocity $\bar{u}_{\bar{z}}$ is uniform and independent in both $\bar{\psi}$ and $\bar{z}$ directions. Thus, the continuity equations can be reduced as 


$$
\frac{\partial \bar{u}}{\partial \bar{z}}=0
$$

The motion is a steady flow which means no variation concerning time and the gravitational acceleration $\bar{g}_{\bar{r}}=\bar{g}_{\bar{\psi}}=\bar{g}_{\bar{z}}=0$ for the horizontal flow [22]. The simplified form in the axial and radial direction of momentum equations can be written as

$$
\begin{aligned}
& \frac{d \bar{p}}{d \bar{z}}=-\frac{1}{\bar{r}} \frac{\partial}{\partial \bar{r}}(\bar{r} \bar{\tau}), \\
& \frac{d \bar{p}}{d \bar{r}}=0,
\end{aligned}
$$

where $d \bar{p} / d \bar{z}$ is the gradient of the stress, $\bar{p}$ is the constant pressure and $\bar{\tau}$ is the shear stress. The constitutive equation of the Bingham-Plastic plastic fluid model as mentioned by [17] is given as:

$$
\frac{d \bar{u}}{d \bar{r}}=\left\{\begin{array}{cl}
-\frac{1}{\eta_{B}}\left(\bar{\tau}-\bar{\tau}_{y}\right), & \text { if } \bar{\tau} \geq \bar{\tau}_{y} \text { and } \bar{r}_{p} \leq \bar{r} \leq \bar{R}(\bar{z}), \\
0, & \text { if } \bar{\tau}<\bar{\tau}_{y} \text { and } 0 \leq \bar{r}<\bar{r}_{p},
\end{array}\right.
$$

where $\bar{\tau}_{y}$ is the yield stress and $\eta_{B}$ is the Bingham-Plastic fluid viscosity coefficient with dimension $M L^{-1} T^{-1}$. Equation (4) displays normal shear flow in the field when $\bar{\tau} \geq \bar{\tau}_{y}$ while solid-like flow takes place when $\bar{\tau}<\bar{\tau}_{y}$. Equations (2) and (4) define the nonlinear system of a differential equation that can be solved under the following boundary constraints for undefined shear stress and velocity distribution

$$
\begin{aligned}
& \bar{\tau} \text { is finite at } \bar{r}=0, \\
& \bar{u}=0 \text { at } \bar{r}=\bar{R}(\bar{z}) .
\end{aligned}
$$

As stated by Rana and Murthy [23], a two-dimensional unsteady convective-diffusion equation is as follows

$$
\frac{\partial \bar{C}}{\partial \bar{t}}=-\bar{u} \frac{\partial \bar{C}}{\partial \bar{z}}+\bar{D}_{m}\left(\frac{1}{\bar{r}} \frac{\partial}{\partial \bar{r}}\left(\bar{r} \frac{\partial \bar{C}}{\partial \bar{r}}\right)+\frac{\partial^{2} \bar{C}}{\partial \bar{z}^{2}}\right),
$$

where $\bar{C}$ is the local solute concentration, $\bar{t}$ is the time variable and $\bar{D}_{m}$ is the molecular diffusion coefficient.

Following Gill et al., [10], the initial and boundary constraints with reference concentration, $\bar{C}_{0}$ are 


$$
\begin{aligned}
& \bar{C}(\bar{r}, \bar{z}, 0)=\left\{\begin{array}{l}
\bar{C}_{0} \text { if }|\bar{z}| \leq \frac{\bar{z}_{s}}{2}, \\
0 \text { if }|\bar{z}|>\frac{\bar{z}_{s}}{2},
\end{array}\right. \\
& \bar{C}(\bar{r}, \infty, \bar{t})=0, \\
& \frac{\partial \bar{C}}{\partial \bar{r}}(0, \bar{z}, \bar{t})=0=\frac{\partial \bar{C}}{\partial \bar{r}}(\bar{R}(\bar{z}), \bar{z}, \bar{t}),
\end{aligned}
$$

where $\bar{z}_{s}$ is the solute length.

\subsection{Geometry of Stenosis}

The dimensional geometry of an overlapping stenosed artery is given by

$$
\bar{R}(\bar{z})=\left\{\begin{array}{cl}
R_{0}-\frac{3 \bar{\delta}}{2 \bar{l}_{0}^{4}}\left[\begin{array}{c}
11(\bar{z}-\bar{d}) \bar{l}_{0}^{3}-47(\bar{z}-\bar{d})^{2} \bar{l}_{0}^{2} \\
+72(\bar{z}-\bar{d})^{3} \bar{l}_{0}-36(\bar{z}-\bar{d})^{4}
\end{array}\right], & \bar{d} \leq \bar{z} \leq \bar{d}+\bar{l}_{0}, \\
R_{0}, & \text { Otherwise, }
\end{array}\right.
$$

where $R_{0}$ is the arterial radius, $\bar{\delta}$ is the stenosis height, $\bar{l}_{0}$ is the stenosis length, $\bar{z}$ is the longitudinal (axial) distance, $\bar{d}$ is the distance of the stenosis from the inlet and $\bar{R}(\bar{z})$ is the stenotic artery radius. Figure 1 indicates the geometry of an overlapping stenosed artery that shows the maximum stenosis height exists at $\bar{d}+\bar{l}_{0} / 3$ and $\bar{d}+2 \bar{l}_{0} / 3$ and $\bar{L}$ is the length of the artery.

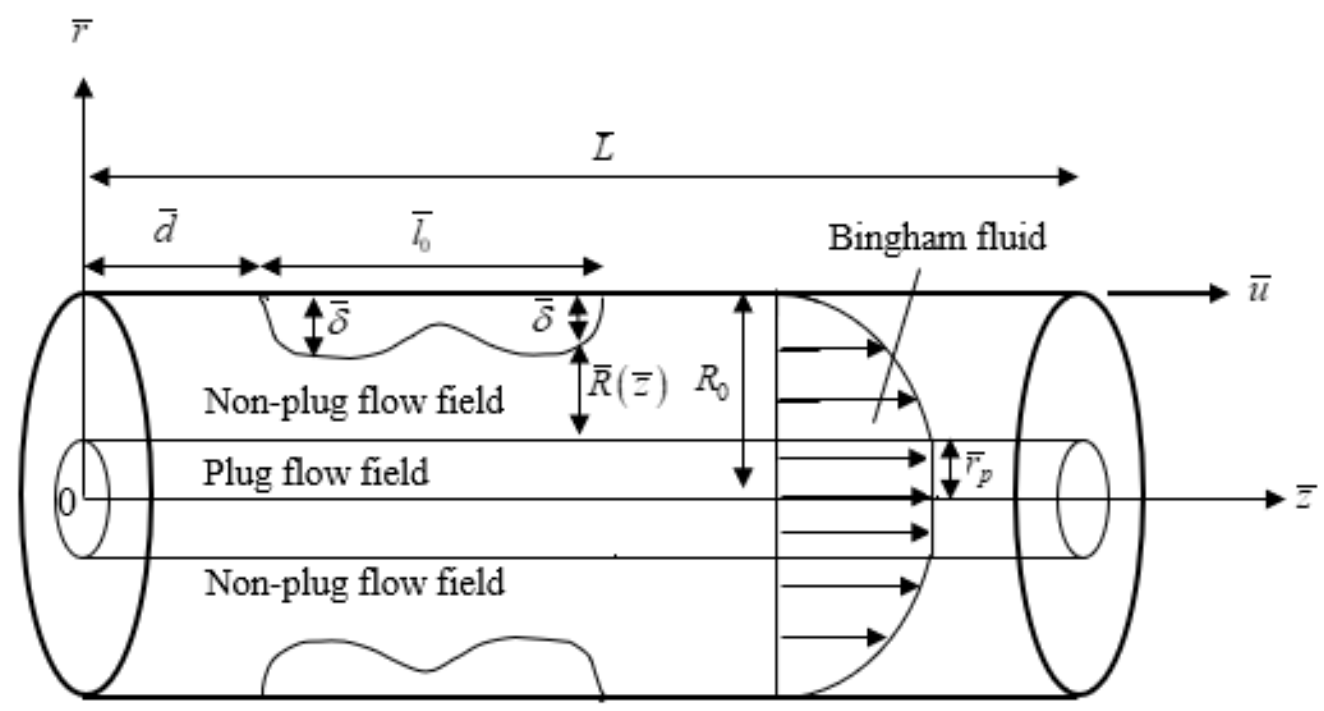

Fig.1. The geometry of an overlapping stenosed artery

\subsection{Non-dimensional Variables}

Introduce some of the non-dimensional variables 


$$
\begin{aligned}
& C=\frac{\bar{C}}{\bar{C}_{0}}, u=\frac{\bar{u}}{\bar{u}_{0}}, u_{+}=\frac{\bar{u}_{+}}{\bar{u}_{0}}, u_{-}=\frac{\bar{u}_{-}}{\bar{u}_{0}}, \bar{u}_{0}=\frac{\bar{R}(\bar{z})^{2}}{\eta_{B}}\left(-\frac{1}{4} \frac{d \bar{p}}{d \bar{z}}\right), u_{m}=\frac{\bar{u}_{m}}{\bar{u}_{0}}, r=\frac{\bar{r}}{R_{0}}, \\
& r_{p}=\frac{\bar{r}_{p}}{R_{0}}, z=\frac{D_{m} \bar{z}}{R_{0}^{2} \bar{u}_{0}}, z_{s}=\frac{D_{m} \bar{z}_{s}}{R_{0}^{2} \bar{u}_{0}}, t=\frac{D_{m} \bar{t}}{R_{0}^{2}} \tau=\frac{\bar{\tau}}{\left(\eta_{B} \bar{u}_{0} / R_{0}\right)}, \tau_{y}=\frac{\bar{\tau}_{y}}{\left(\eta_{B} \bar{u}_{0} / R_{0}\right)}, \\
& R(z)=\frac{\bar{R}(\bar{z})}{R_{0}}, \delta=\frac{\bar{\delta}}{R_{0}}, p=\frac{D_{m} \bar{p}}{\bar{u}_{0} R_{0}\left(\eta_{B} \bar{u}_{0} / R_{0}\right)},
\end{aligned}
$$

where $\bar{u}_{0}$ is the fluid characteristics velocity, $C$ is the solute concentration, $u$ is the velocity, $u_{+}, u_{-}$ is the outer and plug flow velocity, $u_{m}$ is the average velocity, $r$ is the transverse (radial) distance, $r_{p}$ is the radius of the plug core field, $z$ is the longitudinal (axial) distance, $z_{s}$ is the length of solute, $t$ is the time, and $\delta$ is the stenosis height. Using non-dimensional variables in Eq. (12), the corresponding equations and boundary constraints from Eq. (1) until Eq. (11) are as follows, respectively

$$
\begin{aligned}
& \frac{\partial u}{\partial z}=0, \\
& \frac{\partial p}{\partial z}=-\frac{1}{r} \frac{\partial}{\partial r}(r \tau),
\end{aligned}
$$

$\tau$ is finite at $r=0$,

$u=0$ at $r=R(z)$.

$\frac{\partial C}{\partial t}=-u \frac{\partial C}{\partial z}+D_{m}\left(\frac{1}{r} \frac{\partial}{\partial r}\left(r \frac{\partial C}{\partial r}\right)+\frac{\partial^{2} C}{\partial z^{2}}\right)$,

$C(r, z, 0)=\left\{\begin{array}{l}1 \text { if }|z| \leq \frac{z_{s}}{2}, \\ 0 \text { if }|z|>\frac{z_{s}}{2},\end{array}\right.$

$C(r, \infty, t)=0$,

$\frac{\partial C}{\partial r}(0, z, t)=0=\frac{\partial C}{\partial r}(R(z), z, t)$, 


$$
R(z)=\left\{\begin{array}{cc}
1-\frac{3 \delta}{2 l_{0}{ }^{4}}\left[\begin{array}{cc}
11(z-d) l_{0}^{3}-47(z-d)^{2} l_{0}^{2} \\
+72(z-d)^{3} l_{0}-36(z-d)^{4}
\end{array}\right], & d \leq z \leq d+l_{0}, \\
1, & \text { Otherwise }
\end{array}\right.
$$

\subsection{Method of Solution}

Integrating Eq. (2) with respect to under boundary constraint (5) the expression attained for shear stress is

$$
\bar{\tau}=-\frac{\bar{r}}{2} \frac{d \bar{p}}{d \bar{z}}
$$

Substituting Eq. (24) into the Eq. (4) and integrate with respect to $r$ with boundary constraints (6) yields the velocity of the outer and plug flow fields in non-dimensional forms as follows

$$
\begin{aligned}
& u_{+}(r)=\left[\frac{\left(R(z)-r_{p}\right)^{2}-\left(r-r_{p}\right)^{2}}{R(z)^{2}}\right], \quad \text { if } \tau \geq \tau_{y} \text { and } r_{p} \leq r \leq R(z), \\
& u_{-}\left(r_{p}\right)=\left[\frac{\left(R(z)-r_{p}\right)^{2}}{R(z)^{2}}\right], \quad \text { if } \tau<\tau_{y} \text { and } 0 \leq r<r_{p} .
\end{aligned}
$$

The non-dimensional form of the mean velocity, $u_{m}$ is

$$
u_{m}=\left(\frac{1}{2}-\frac{2}{3} \frac{r_{p}}{R(z)}+\frac{1}{6} \frac{r_{p}^{4}}{R(z)^{4}}\right)
$$

According to Gill [9] the solution of Eq. (19) with the constraints (20), (21) and (22) is formulated as a series expansion involving about the mean concentration to describe the local concentration and is displayed as

$$
C(r, z, t)=C_{m}\left(z_{1}, t\right)+\sum_{i=R(z)}^{\infty} f_{i}(r, t) \frac{\partial^{i} C_{m}\left(z_{1}, t\right)}{\partial z_{1}^{i}}
$$

where $C_{m}\left(z_{1}, t\right)=2 \int_{0}^{R(z)} C(r, z, t) r d r$ is the mean concentration, $f_{i}(r, t)$ is the dispersion function and $z_{1}=z-u_{m} t$ is a new axial coordinate moves with the average velocity. Gill et al., [10] mentioned that the process of distributing $C_{m}(z, t)$ is diffusive from the beginning of time and hence, the generalized dispersion model as suitable functions of time $t$ is

$$
\frac{\partial C_{m}}{\partial t}=\sum_{i=1}^{\infty} K_{i}(t) \frac{\partial^{i} C_{m}}{\partial z_{1}^{i}}\left(z_{1}, t\right)
$$


where $K_{1}(t)$ is the coefficient of longitudinal convection and $K_{2}(t)$ is the coefficient of longitudinal diffusion. Since, $K_{2}(t)$ expresses the whole dispersion process in terms of the simple diffusion process in axial direction $z_{1}$, it is also called as effective longitudinal diffusivity. The initial and boundary constraints $f_{i}$ can be obtained from eq. (20)-(22) and (28) as follows

$$
\begin{aligned}
& f_{i}(r, 0)=0, \\
& \frac{\partial f_{i}}{\partial r}(0, t)=0=\frac{\partial f_{i}}{\partial r}(R(z), t) .
\end{aligned}
$$

The solvability condition is

$$
\int_{0}^{R(z)} f_{i} r d r=0
$$

Jaafar et al., [24] stated that the function $f_{1}$ in the Eq. (28) is prominent to measure $C(r, z, t)$. The solution to the non-homogenous parabolic differential equations can be separated into two following parts

$$
f_{1}(r, t)=f_{1 s}(r)+f_{1 t}(r, t)
$$

where $f_{1 s}(r)$ is the dispersion function in the steady-state and $f_{1 t}(r, t)$ is the dispersion function in the transient state which exemplified the time-dependent nature of the solute dispersion. By substituting Eq. (33) into the constraints (30) and (31) then grouping $f_{1 s}(r)$ and $f_{1 t}(r, t)$, the initial constraints of $f_{1 t}(r, t)$ is

$$
f_{1 t}(r, 0)=-f_{1 s}(r)
$$

and the boundary constraints of $f_{1 s}(r)$ and $f_{1 t}(r, t)$ are

$$
\begin{aligned}
& \frac{d f_{1 s}}{d r}(r=0)=0=\frac{d f_{1 s}}{d r}(r=R(z)), \\
& \frac{\partial f_{1 t}}{\partial r}(0, t)=0=\frac{\partial f_{1 t}}{\partial r}(R(z), t) .
\end{aligned}
$$

The differential equation of dispersion function at the steady-state in a plug flow field is

$$
\frac{1}{r} \frac{d}{d r}\left(r \frac{d f_{1 s_{-}}}{d r}\right)-\left(u_{-}-u_{m}\right)=0, \quad \text { if } 0 \leq r<r_{p}
$$

and in the outer flow field becomes 
$\frac{1}{r} \frac{d}{d r}\left(r \frac{d f_{1 s_{+}}}{d r}\right)-\left(u_{+}-u_{m}\right)=0, \quad$ if $r_{p} \leq r \leq R(z)$.

Solving Eq. (37) numerically subject to the boundary constraint (35), the steady dispersion function in the plug flow field $f_{1 s_{-}}(r)$ and outer field $f_{1 s_{+}}(r)$ are, respectively

$$
\begin{aligned}
& f_{1 s_{-}}(r)=\left(\frac{1}{8}-\frac{1}{24} \frac{r_{p}^{4}}{R(z)^{4}}+\frac{1}{4} \frac{r_{p}{ }^{2}}{R(z)^{2}}-\frac{1}{3} \frac{r_{p}}{R(z)}\right) r^{2}+S_{1}, \\
& f_{1 s_{+}}(r)=\left(\frac{1}{8}-\frac{1}{24} \frac{r_{p}^{4}}{R(z)^{4}}-\frac{1}{3} \frac{r_{p}}{R(z)}\right) r^{2}-\frac{r^{4}}{16 R(z)^{2}}+\frac{2 r^{3} r_{p}}{9 R(z)^{2}}+\frac{13 r_{p}^{4}}{144 R(z)^{2}} \\
&+\frac{r_{p}^{4}}{12 R(z)^{2}} \log \left(\frac{r}{r_{p}}\right)+S_{1},
\end{aligned}
$$

where $S_{1}$ is delineated as

$$
S_{1}=-\frac{r_{p}{ }^{6}}{120 R(z)^{4}}-\frac{r_{p}{ }^{4}}{36 R(z)^{2}}+\frac{7 r_{p} R(z)}{90}-\frac{R(z)^{2}}{24}+\frac{r_{p}{ }^{4}}{12 R(z)^{2}} \log \left(\frac{r_{p}}{R(z)}\right)
$$

The dispersion function in the transient state, $f_{1 t}(r, t)$ can be solved using the method of separation of variable and Bessel function subject to the boundary constraints (34) and (36). The solution $f_{1 t}(r, t)$ is computed numerically using Simpson's 3/8 rule and is obtained as

$$
f_{1 t}(r, t)=\sum_{m=1}^{\infty} A_{m} e^{-\lambda_{m}^{2} t} J_{0}\left(\lambda_{m} r\right)
$$

where

$$
A_{m}=-\frac{\int_{0}^{R(z)} J_{0}\left(\lambda_{m} r\right) f_{1 s}(r) r d r}{\int_{0}^{R(z)} J_{0}^{2}\left(\lambda_{m} r\right) r d r}=-\frac{2}{J_{0}^{2}\left(\lambda_{m}\right)} \int_{0}^{R(z)} J_{0}\left(\lambda_{m} r\right) f_{1 s}(r) r d r,
$$

and the eigenvalues $\lambda_{m}$ are the roots of the equation $J_{1}(r)=0 . J_{0}$ and $J_{1}$ denotes Bessel's functions of the first kind of order zero and one respectively.

\section{Results and Discussions}

The effect of varying stenosis height and plug core radius on the steady dispersion function, unsteady dispersion function, dispersion function, normalized velocity and mean velocity is analyzed. The range of values of parameters used in this study are as follows: $r_{p}: 0-0.2, \delta: 0.01-0.3, l_{0}: 3, d: 2$ and $z: 4[20,24,25]$. In this study, Mathematica software is used to generate the results and data for 
validation and comparison purposes. The present results of Bingham fluid with the impact of the stenosis height and plug core radius on the solute dispersion problem are validated with $[26,27]$. The validation of steady dispersion function $f_{1 s}$, unsteady dispersion function $f_{1 t}$ and dispersion function $f_{1}$ when $\delta=0, t=0.1$ and $r_{p}=0.1$ are illustrated in Figure 2 and Figure 3. It is elucidated that the aforesaid figures are in good agreement with $[26,27]$. In the present study, the geometry of the stenosed artery $\mathrm{R}(\mathrm{z})$ and $\mathrm{n}$ is equal to one in the absence of stenosis and the special case for HerschelBulkley fluid artery $R(z)$ and $n$ is equal to one in the absence of stenosis and the special case for Herschel-Bulkley fluid when $n=1$ ascribed for Bingham fluid model are used for validation. It can be seen that $f_{1 s}$ and $f_{1}$ decreases at the center of an artery meanwhile $f_{1 t}$ delineates a reverse behavior. As observed by Patel and Sirs [28], one of the factors that influence the dispersion of solutes in blood flow is the flexing of red blood cells and their migration to the core.

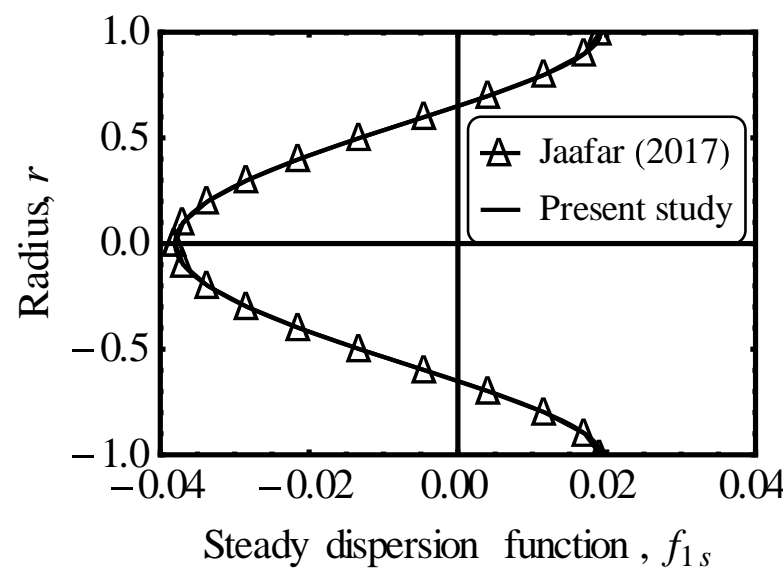

(a)

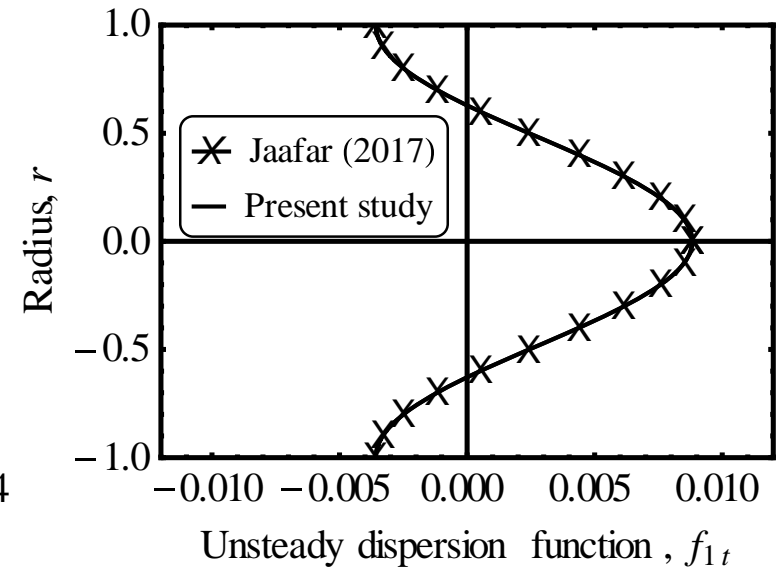

(b)

Fig. 2. Validation of (a) steady dispersion function and (b) unsteady dispersion function with a radius $r$ when $\delta=0, R(z)=1, n=1, r_{p}=0.1$ and $t=0.1$.

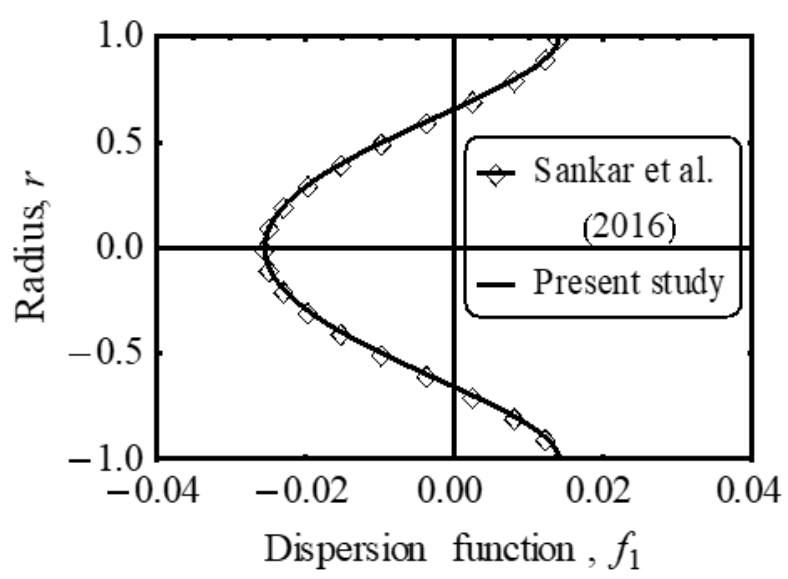

Fig. 3. Validation of dispersion function with a radius $r$ when $\delta=0, R(z)=1, n=1, r_{p}=0.1$ and $t=0.1$.

Figure 4 described the variation of steady and unsteady dispersion function with a radius $r$ for different values of stenosis height, $\delta$ when $l_{0}=3, d=2, z=4$ and $r_{p}=0.1$. The effect of stenosis height is prominent to determine the size of stenosis. As the stenosis height increase, the stenosed artery becomes narrower and affects the normal flow of blood to the artery. It can be seen that when the stenosis height increases in the range $\delta=0.01,0.02,0.03,0.04$ and 0.05 , the steady dispersion 
function $f_{1 s}$ of the solute decreases. A reverse behavior is displayed for the dispersion function at the unsteady-state for aforesaid rheological parameters. When the stenosis height increases, the unsteady dispersion function $f_{1 t}$ of the solute also increases.

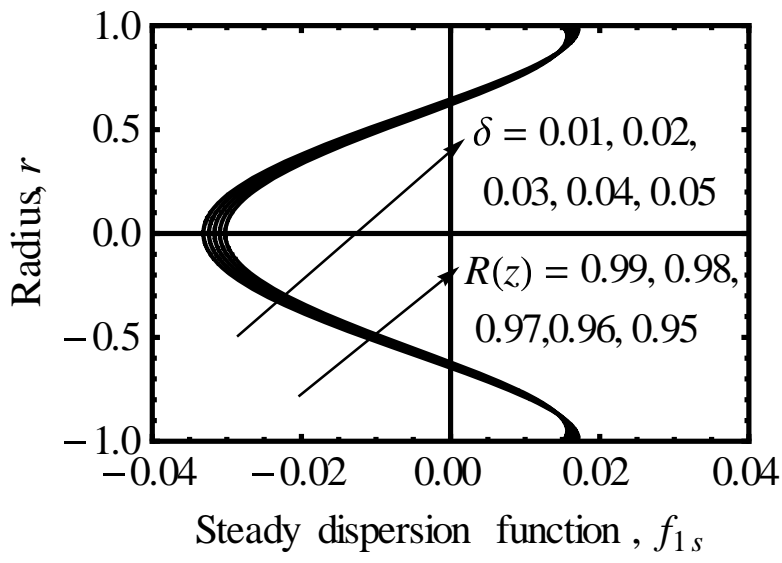

(a)

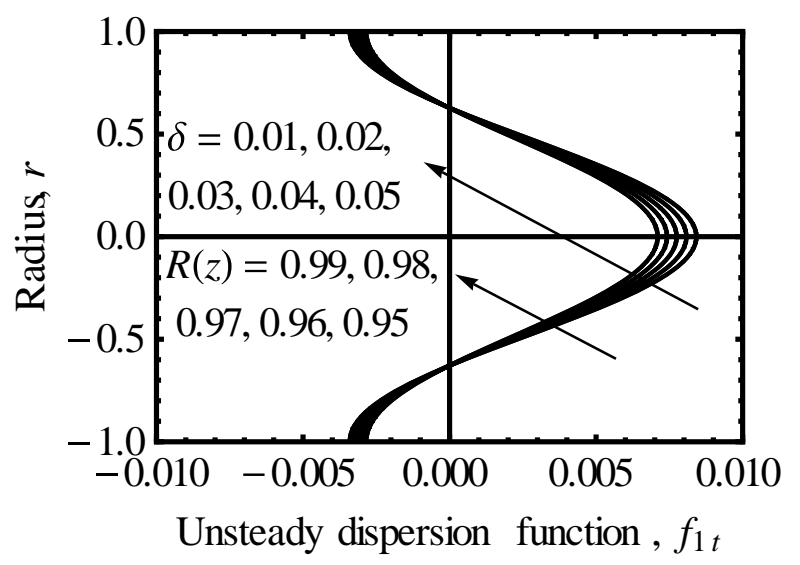

(b)

Fig. 4. Variation of (a) steady dispersion function and (b) unsteady dispersion function with a radius $r$ for different values of stenosis height, $\delta$ when $l_{0}=3, d=2, z=4$ and $r_{p}=0.1$.

Figure 5 depicts the variation of dispersion function with a radius $r$ for different values of stenosis height, $\delta$ when $l_{0}=3, d=2, z=4$ and $r_{p}=0.1$. It shows that as the stenosis height increase, the radius of the stenosed artery decreases in the range $R(z)=0.99,0.98,0.97,0.96$ and 0.95 . The dispersion function inhibits the same pattern as steady dispersion function whereby an increase in the stenosis height indicates a decrease in the dispersion function. As mentioned by Dash et al., [29], $f_{1}$ is aimed to mitigate the concentration at the plug core and also to magnify it close to the wall.

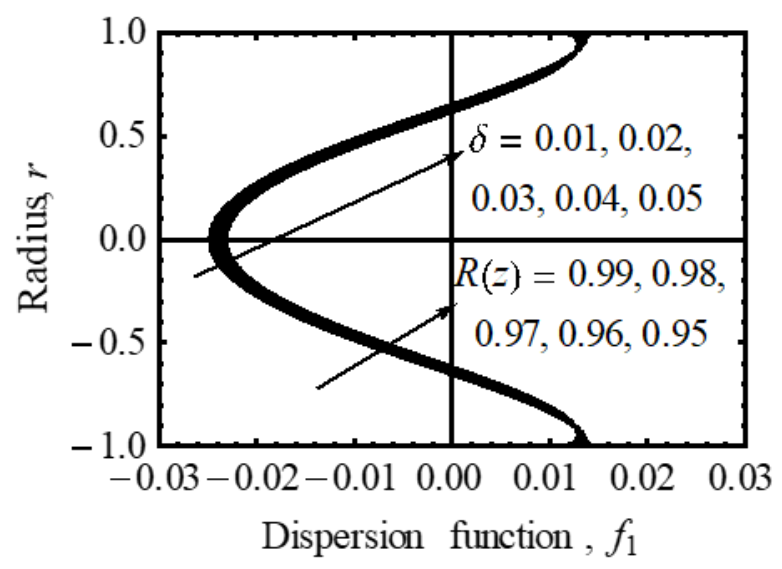

Fig. 5. Variation of dispersion function with a radius $r$ for different values of stenosis height, $\delta$ when $l_{0}=3, d=2, z=4$ and $r_{p}=0.1$.

The effect of varying time $t$ on the dispersion function with a radius $r$ when $\delta=0.01, l_{0}=3$, $d=2, z=4$ and $R(z)=0.99$ at (a) $r_{p}=0$ (b) $r_{p}=0.05$ (c) $r_{p}=0.1$ and (d) $r_{p}=0.2$ is ascribed in Figure 6 . It is portrayed that the dispersion function $f_{1}$ decreases as the plug core radius $r_{p}$ and time $t$ increase in the range of $r=-0.65$ to $r=0.65$ and displayed an opposite behavior in the rest of the range because $f_{1}$ is independent of $t$ at the center. There is a high amount of red blood cells at the 
center of the artery but no influences near the wall. As time $t$ increases from $t=0.05,0.1,0.15,0.2$ and $0.5, f_{1}$ approaches its steady-state value $f_{1 s}$ at $t=0.5$. When the plug core radius $r_{p}=0$, the dispersion function corresponds to the Newtonian fluid and the magnitude $f_{1}$ is maximum which is in agreement with the observation of [10]. It can be remarked from these figures that the dispersion function $f_{1}$ passes through a common point for all time $t$ and all values of the plug core radius $r_{p}$.

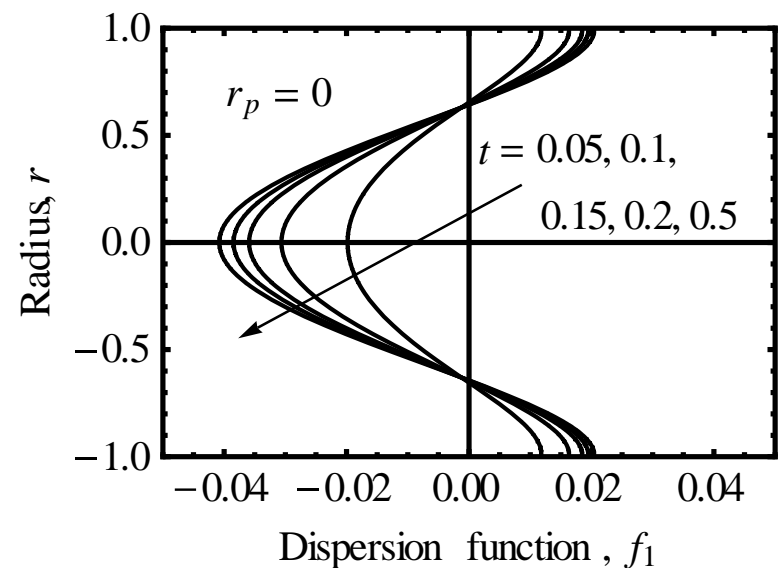

(a)

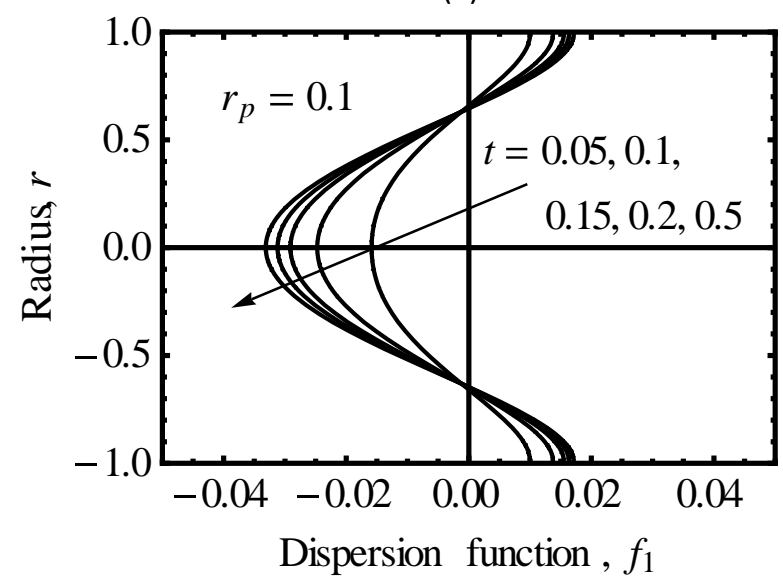

(c)

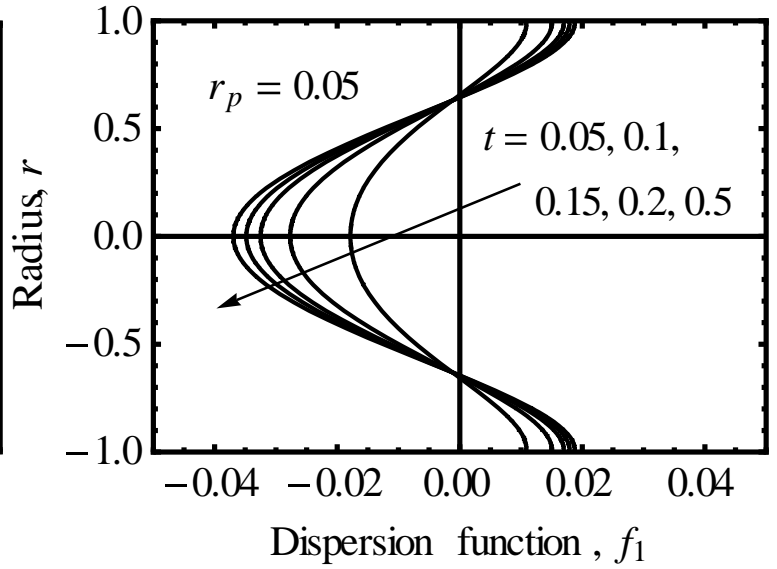

(b)

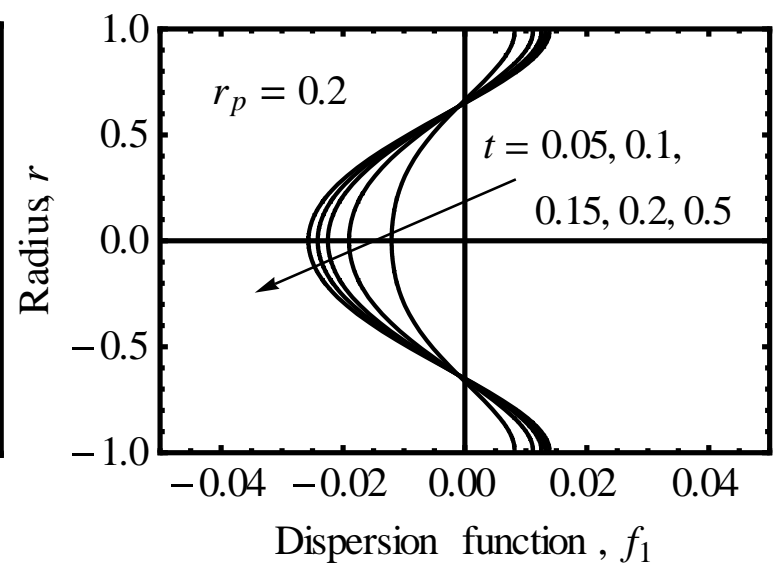

(d)

Fig. 6. Variation of dispersion function with a radius $r$ for different values of time $t$ when $\delta=$ $0.01, l_{0}=3, d=2, z=4$ and $R(z)=0.99$ at (a) $r_{p}=0$ (b) $r_{p}=0.05$ (c) $r_{p}=0.1$ and (d) $r_{p}=$ 0.2 .

The estimates of normalized velocity and mean velocity in the dispersion of solutes when $l_{0}=$ $3, d=2, z=4$ and $r_{p}=0.1$ for the canine vascular systems are delineated in Table 1 . The physiological data for different types of arteries is ascribed by [25]. It is depicted that as stenosis height increases, the normalized velocity profiles and mean velocity decrease marginally. The normalized velocity decreases due to the diffusion of solute to the wall of the artery. It can be seen that arteriole having diameter has the highest normalized velocity and mean velocity however as the stenosis height increase, the normalized velocity and mean velocity monotonically decrease. 


\section{Table 1}

Estimates of normalized velocity and mean velocity in the dispersion of solutes when $r_{p}=0.01, l_{0}=3, d=2$ and $z=4$ for the canine vascular systems

\begin{tabular}{llllllll}
\hline Artery type & Radius & \multicolumn{3}{c}{ Normalized velocity } & \multicolumn{5}{c}{ Mean velocity } \\
\cline { 2 - 8 } & $\left(\times 10^{-2} \mathrm{~m}\right)$ & $\delta=0.1$ & $\delta=0.2$ & $\delta=0.3$ & $\delta=0.1$ & $\delta=0.2$ & $\delta=0.3$ \\
\hline Aorta & 1.0 & 1.986 & 1.983 & 1.981 & 0.493 & 0.492 & 0.490 \\
Femoral & 0.5 & 1.967 & 1.956 & 1.934 & 0.483 & 0.478 & 0.467 \\
Carotid & 0.4 & 1.956 & 1.934 & 1.810 & 0.478 & 0.467 & 0.433 \\
Coronary & 0.15 & 1.744 & 1.673 & 1.590 & 0.367 & 0.334 & 0.307 \\
Arteriole & 0.008 & 2.147 & 2.070 & 2.046 & 0.572 & 0.535 & 0.523 \\
\hline
\end{tabular}

\section{Conclusion}

The current study aims to examine the impact of non-Newtonian blood rheology and stenosis height on the solute dispersion process in the cardiovascular system. Some of the significant findings are summarized below

i. The steady dispersion function decreases at the center of the artery as the stenosis height increase from $\delta=0.01,0.02,0.03,0.04$ and 0.05 , however, a reverse behavior is shown for the unsteady dispersion function.

ii. It is noted that there is a high amount of red blood cells at the center of the artery but no influences near the wall hence, the dispersion function decreases at the center of the artery with an increase in the plug core radius when $r_{p}=0,0.05,0.1,0.2$ and time, $t=$ $0.05,0.1,0.15,0.2$ and 0.5 .

iii. The non-Newtonian fluid behavior which is the yield stress is related to the nonNewtonian nature of the fluid and with an increase in the yield stress, the viscosity of the blood increases. The non-Newtonian fluid behavior and stenosis height affect significantly the dispersion of solutes in a solvent (blood).

\section{Acknowledgement}

The authors would like to acknowledge the Ministry of Higher Education and Research Management Centre, Universiti Teknologi Malaysia for the financial support and the authors are also grateful for the support and hospitality of the Sydney Mathematical Research Institute (SMRI) and Griffith university during preparing this work. This work was supported by the Ministry of Higher Education under Fundamental Research Grant Scheme FRGS/1/2019/STG06/UTM/02/21 and Research Management Centre, Universiti Teknologi Malaysia (UTM) under UTM Fundamental Research PY/2019/01623-Q.J130000.2554.21H48.

\section{References}

[1] World Health Organization 2020 (2019, November) Cardiovascular Diseases (CVDs) Retrieved from the World Health Organization website: https://www.who.int/news-room/fact-sheets/detail/the-top-10-causes-of-death.

[2] Gholipour, Alireza, Mergen H. Ghayesh, Anthony Zander, and Rajiv Mahajan. "Three-dimensional biomechanics of coronary arteries." International Journal of Engineering Science $130 \quad$ (2018): 93-114. https://doi.org/10.1016/i.ijengsci.2018.03.002

[3] Shah, Sapna Ratan. "Study of modified Casson's fluid model in modeled normal and stenotic capillary-tissue diffusion phenomena." International Journal of Computational Engineering \& Management 11 (2011): 51-57.

[4] Mishra, Shailesh, and S. U. Siddiqui. "A mathematical model for flow and diffusion through stenotic capillary-tissue exchange system." Journal of Science and Technology 4, no. 1 (2015): 299-315.

[5] Majee, Sreeparna, and G. C. Shit. "Modeling and simulation of blood flow with magnetic nanoparticles as carrier for targeted drug delivery in the stenosed artery." European Journal of Mechanics-B/Fluids 83 (2020): 42-57. https://doi.org/10.1016/j.euromechflu.2020.04.004 
[6] Sharifzadeh, Bahador, Rasool Kalbasi, Mehdi Jahangiri, Davood Toghraie, and Arash Karimipour. "Computer modeling of pulsatile blood flow in elastic artery using a software program for application in biomedical engineering." Computer methods and programs in biomedicine $192 \quad$ (2020): 105442. https://doi.org/10.1016/j.cmpb.2020.105442

[7] Taylor, Geoffrey Ingram. "The dispersion of matter in turbulent flow through a pipe." Proceedings of the Royal Society of London. Series A. Mathematical and Physical Sciences 223, no. 1155 (1954): 446-468. https://doi.org/10.1098/rspa.1953.0139

[8] Aris, Rutherford. "On the dispersion of a solute in a fluid flowing through a tube." Proceedings of the Royal Society of London. Series A. Mathematical and Physical Sciences 235, no. 1200 (1956): 67-77. https://doi.org/10.1098/rspa.1956.0065

[9] Gill, WN-Na5233. "A note on the solution of transient dispersion problems." Proceedings of the Royal Society of London. Series A. Mathematical and Physical Sciences 298, no. 1454 (1967): 335-339. https://doi.org/10.1098/rspa.1967.0107

[10] Gill, W. N., and R. Sankarasubramanian. "Exact analysis of unsteady convective diffusion." Proceedings of the Royal Society of London. A. Mathematical and Physical Sciences 316, no. 1526 (1970): 341-350. https://doi.org/10.1098/rspa.1970.0083

[11] Ananthkrishnan, V., W. N. Gill, and A. J. Barduhan. "Laminar dispersion in capillaries: part I." Math. Anal. AICHE J 11 (1965): 1063-1072. https://doi.org/10.1002/aic.690110620

[12] Shahzadi, Iqra, and S. Bilal. "A significant role of permeability on blood flow for hybrid nanofluid through bifurcated stenosed artery: drug delivery application." Computer methods and programs in biomedicine 187 (2020): 105248. https://doi.org/10.1016/i.cmpb.2019.105248

[13] Haghighi, Ahmad Reza, Nooshin Aliashrafi, and Mohammad Shahbazi Asl. "An implicit approach to the micropolar fluid model of blood flow under the effect of body acceleration." Mathematical Sciences 14, no. 3 (2020): $269-277$. https://doi.org/10.1007/s40096-020-00340-x

[14] Das, Prosanjit, and Prashanta Kumar Mandal. "Solute dispersion in Casson fluid flow through a stenosed artery with absorptive wall." Zeitschrift für angewandte Mathematik und Physik 71 (2020): 1-24. https://doi.org/10.1007/s00033-020-01322-8

[15] Chakravarty, S., and PK14035210854 Mandal. "A nonlinear two-dimensional model of blood flow in an overlapping arterial stenosis subjected to body acceleration." Mathematical and computer modelling 24, no. 1 (1996): 43-58. https://doi.org/10.1016/0895-7177(96)00079-9

[16] Ahmed, Ashfaq, and Sohail Nadeem. "Biomathematical study of time-dependent flow of a Carreau nanofluid through inclined catheterized arteries with overlapping stenosis." Journal of Central South University 24, no. 11 (2017): 2725-2744. https://doi.org/10.1007/s11771-017-3685-4

[17] Bhatnagar, Amit, and R. K. Shrivastav. "Analysis of flow characteristics through an artery with time dependent overlapping stenosis." In 2014 International Conference on Advances in Computing, Communications and Informatics (ICACCI), pp. 462-467. IEEE, 2014. https://doi.org/10.1109/ICACCI.2014.6968507

[18] Hossain, Khan Enaet, and Md Mohidul Haque. "Influence of magnetic field on chemically reactive blood flow through stenosed bifurcated arteries." In AIP Conference Proceedings, vol. 1851, no. 1, p. 020012. AIP Publishing LLC, 2017. https://doi.org/10.1063/1.4984641

[19] Freidoonimehr, Navid, Rey Chin, Anthony Zander, and Maziar Arjomandi. "An experimental model for pressure drop evaluation in a stenosed coronary artery." Physics of Fluids 32, no. 2 (2020): 021901. https://doi.org/10.1063/1.5139701

[20] Miah, Md Abdul Karim, Shorab Hossain, and Sayedus Salehin. "Effects of Severity and Dominance of Viscous Force on Stenosis and Aneurysm During Pulsatile Blood Flow Using Computational Modelling." (2021). https://doi.org/10.37934/cfdl.12.8.3554

[21] Swarup, S. " Fluid Dynamics." (1991).

[22] White, Frank M. "Fluid Mechanics." (2011)..

[23] Rana, Jyotirmoy, and P. V. S. N. Murthy. "Unsteady solute dispersion in Herschel-Bulkley fluid in a tube with wall absorption." Physics of Fluids 28, no. 11 (2016): 111903. https://doi.org/10.1063/1.4967210

[24] Al-Azawy, Mohammed Ghalib, Saleem Khalefa Kadhim, and Azzam Sabah Hameed. "Newtonian and NonNewtonian Blood Rheology Inside a Model of Stenosis." CFD Letters 12, no. 11 (2020): 27-36. https://doi.org/10.37934/cfdl.12.11.2736

[25] Sankar, D. S., and Atulya K. Nagar. "Nonlinear fluid models for biofluid flow in constricted blood vessels under body accelerations: A comparative study." Journal of Applied Mathematics 2012 (2012). https://doi.org/10.1155/2012/950323

[26] Sankar, D. S., Nurul Aini Binti Jaafar, and Yazariah Yatim. "Nonlinear analysis for shear augmented dispersion of solutes in blood flow through narrow arteries." Journal of Applied Mathematics 2012 (2012). 
[27] Jaafar, Nurul Aini, Yazariah Mohd Yatim, and D. S. Sankar. "Mathematical analysis for unsteady dispersion of solute with chemical reaction in blood flow." In AIP Conference Proceedings, vol. 1750, no. 1, p. 030033. AIP Publishing LLC, 2016. https://doi.org/10.1063/1.4954569

[28] Patel, I. C., and J. A. Sirs. "Dispersion of solutes during blood flow through curved tubes." Medical and Biological Engineering and Computing 21, no. 2 (1983): 113-118. https://doi.org/10.1007/BF02441524

[29] Dash, R. K., G. Jayaraman, and K. N. Mehta. "Shear augmented dispersion of a solute in a Casson fluid flowing in a conduit." Annals of Biomedical Engineering 28, no. 4 (2000): 373-385. https://doi.org/10.1114/1.287 